\title{
Acute Type A Aortic Dissection: Pre-Operative Clinical and Radiological Predictors of Outcome
}

\author{
AHMED ELSHARKAWY, M.D. \\ The Department of Cardiothoracic Surgery, Faculty of Medicine, Cairo University
}

\begin{abstract}
Background: Acute type A aortic dissection is a surgical emergency associated with high morbidity and mortality and is still a surgical challenge. The aim of our work is to evaluate the impact of pre-operative clinical status of different malperfusion symptoms (according to Penn classification) and radiological findings on surgical outcome of acute type A dissection patients.
\end{abstract}

Aim of Study: Is to report our experience in surgical management of acute type A dissection patients and to determine the impact of pre-operative ischaemia (by Penn class) on post-operative outcome.

Patients and Methods: Between January 2012 and December 2014, 30 patients (23 males, 7 females) with acute type A aortic dissection were operated upon at Kasr Al-Aini Cardiac Surgery Unit, Cairo University. All patients presented with severe chest pain and pulse deficit. Their age ranged from 30 to 70 years. 2 patients had bicuspid aortic valve and 8 patients had Marfan syndrome. 3 patients had acute lower limb ischemia, 6 had renal malperfusion and 4 had massive pericardial effusion. All patients had pre-operative multislice CT aortography with 4 patients showing extensive arch tears and 4 patients had dissected coronary arteries on multislice CT of the coronaries. 13 patients had supracoronary conduit replacement of ascending aorta, 13 had modified Bentall operation, and 4 had valve-sparing (David) operation. All patients had circulatory arrest at moderate hypothermia and cerebral protection for open repair of the dissected aortic arch.

Results: The mean cross clamp time was $145 \pm 25$ minutes, and total circulatory arrest time was $25 \pm 10$ minutes. Patients with pre-operative malperfusion had more prolonged and complicated post-operative stay. There was $5(17 \%)$ postoperative mortality: 1 due to massive bleeding, 2 due to prolonged ventilation following stroke, 1 due to hepato-renal failure and 1 due to myocardial failure.

Conclusion: Surgical treatment of acute type A aortic dissection is challenging and requires a well-planned strategy to get optimum outcome.

Dissection patients with malperfusion syndromes, circumferential arch tears, and dissected coronaries carry higher risk of mortality.

Correspondence to: Dr. Ahmed El-Sharkawy, E-Mail: ahmedsharkawy.cts@hotmail.com
Key Words: Malperfusion - Dissection - Circulatory arrest.

\section{Introduction}

ACUTE Type A Aortic Dissection (ATAAD) is the most serious aortic emergency and is associated with a high mortality risk, which increases after onset of symptoms. Left untreated, it is highly lethal with a mortality rate of $8 \%$ within the first 6 hours, $13 \%$ within 12 hours, $21 \%$ within 24 hours, and $74 \%$ in the first 2 weeks. This aggressive natural history makes prompt surgical intervention imperative, replacing intimal dissection of the ascending aorta and/or arch by a prosthetic graft and restoring blood flow into the collapsed true lumen $[\mathbf{1 , 2}]$.

In patients with acute type A aortic dissection, early post-operative outcomes are largely influenced by pre-operative patient status, particularly haemodynamic shock, localized or generalized organ ischaemia and malperfusion syndromes. Such states are reflected in the recent Penn classification [3].

Penn classification: This classification system has been validated by Olsson and colleagues in 2011 and has shown merit in predicting ATAADrelated in-hospital mortality by addressing the impact of pre-operative malperfusion and ischaemia at presentation on post-operative outcome [4]

Patients are classified into four distinct subsets (Penn classes):

I- ATAAD Class A: Absence of branch vessel malperfusion or circulatory collapse.

II- ATAAD Class B: Branch vessel malperfusion with ischaemia (renal, cerebral, visceral, limb).

III- ATAAD Class C: Circulatory collapse (massive tamponade with or without coronary involvement). 
IV- ATAAD Class BC: Branch vessel malperfusion and circulatory collapse.

Penn class B and C patients carry worse outcome than Penn A.

\section{The aim of this study:}

Is to report our experience in surgical management of acute type A dissection patients and to determine the impact of pre-operative ischaemia (by Penn class) on post-operative outcome.

\section{Patients and Methods}

This is a retrograde observational study carried out during the period between January 2012 and December 2014 in Cardiac Surgery Unit, Cairo University. We reviewed the records of 30 consecutive patients who had ATAAD surgery by our team during the period of study. All were operated on emergency basis. The demographic and preoperative data of these patients are shown in (Table 1).

\section{Pre-operative preparation:}

A detailed assessment of all patients was done pre-operatively and included full history taking and laboratory investigations especially those related to malperfusion, namely serum creatinine, liver funcyions, and cardiac enzymes. Transthoracic or trans-oesophageal echocardiography routinely done, Multi-Slice Computed Tomography (MSCT) scan to assess the extent of dissection of the aorta. In patients showing elevated serum creatinine, magnetic resonance angiography of the aorta was done as pre-operative imaging.

On MSCT, the more the true lumen is collapsed, the more the malperfusion symptoms are clinically evident.
Fig. (1): MSCT for patient with TAAD showing extensive circumferential arch tear (a) and dissected right coronary artery (b).
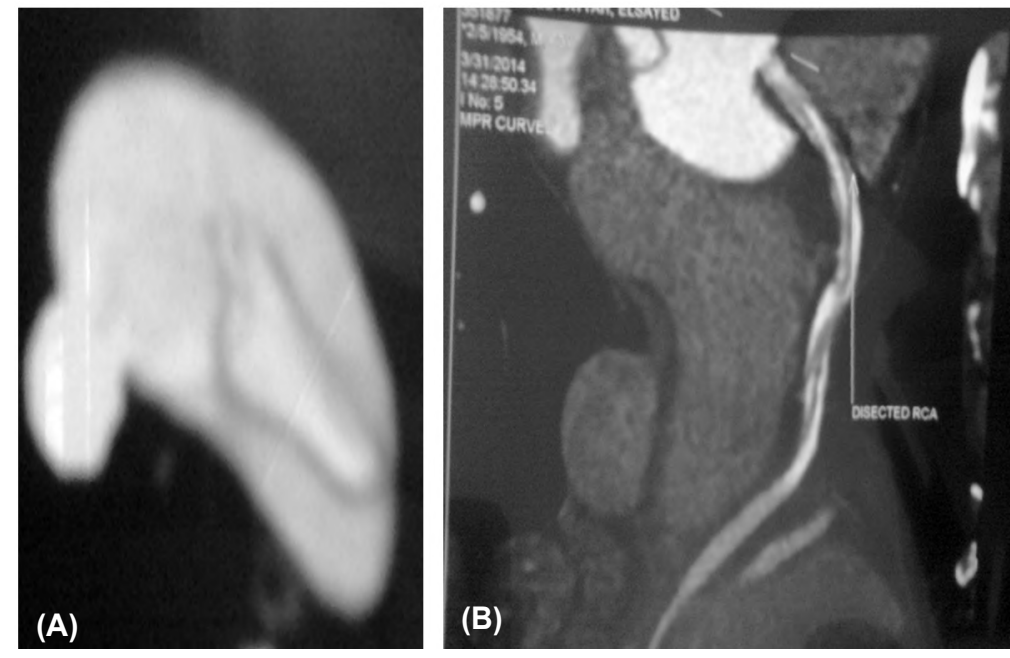

\section{Surgical technique:}

All acute dissection patients are operated on emergency basis at our institute, within 2 to 4 hours of presentation to the emergency room. Special considerations required for all patients include proper pre-operative imaging, proper myocardial protection, proper Cardio-Pulmonary Bypass (CPB) and perfusion strategy.

Anesthetic management includes bilateral radial arterial line monitoring as well as intra-operative trans-esophageal echocardiography. Thiopental 2 gm, steroids, ice packs around the patient head were used during period of circulatory arrest for brain protection.

Cannulation sites for Cardiopulmonary Bypass (CPB): Arterial inflow was done through the femoral artery in 5 patients $(16.6 \%)$ and through the axillary artery in 25 patients ( $83.3 \%)$. Venous return was via the right atrium in all patients.

All patients were subjected to standard median sternotomy, careful dissection around the ascending aorta till arch vessels was done before giving heparin. Myocardial protection was obtained using ante-grade cold crystalloid intermittent cardioplegia every 20-30 minutes and topical ice slush in all case.

Root surgery and ascending aortic replacement (Table 2): The decision making and selection of surgical technique was based on the degree of preoperative aortic valve regurgitation, tissue quality, and whether the coronary Ostia are affected by the intimal tear or not. 13 patients had supracoronary conduit replacement of ascending aorta, $13 \mathrm{had}$ modified Bentall operation, and 4 had valve-sparing (David 1) operation. 
Mode of coronary reimplantation: Direct button reattachement was needed in 11 patients, modified cabrol technique using $8 \mathrm{~mm}$ graft in 3 patients, and Saphenous Vein Bypass Graft (SVG) to the distal Right Coronary Artery (RCA) in 3 patients with dissected coronary ostium.

Circulatory arrest for arch reconstruction: All patients had circulatory arrest under moderate hypothermia (26 degree) for around 20 to $30 \mathrm{~min}$ utes, and cerebral protection for open repair of the dissected aortic arch (hemi arch replacement).

Mode of cerebral perfusion: Ante grade cerebral perfusion in 25 patients through right axillary artery and retrograde perfusion through selective cannulation and snaring of superior vena cava in 5 patients.

Statistical analysis: Continuous variables were expressed as mean and standard deviation $(\mathrm{X} \pm$ $\mathrm{SD})$, and were analyzed by using the unpaired $t$ test. Categorical variables were expressed as number and percentage (No. \& \%). A $p$-value of less than $0.05^{*}$ was considered statistically significant.

\section{Results}

Pre-operative data: This is a retrospective observational study carried on 30 patients with reoperation on the ATAAD, during the period between 2012 and 2014. The demographic and pre-operative clinical characters of these patients are shown in (Table 1).

Table (1): Patients' demographics \& pre-operative clinical characters.

\begin{tabular}{|c|c|}
\hline & $\begin{array}{c}\text { Number (\%) OR } \\
\text { Mean } \pm \text { SD }\end{array}$ \\
\hline \multicolumn{2}{|l|}{ Demographic: } \\
\hline Age (range-years) & $30-70(50 \pm 20)$ \\
\hline Male gender & $23(76.6 \%)$ \\
\hline Marfan syndrome & $8 \quad(26.6 \%)$ \\
\hline Bicuspid Aortic valve & $2(6.6 \%)$ \\
\hline Previous cardiac operations & - \\
\hline Hypertension & $27(90 \%)$ \\
\hline \multicolumn{2}{|c|}{ Pre-operative Malperfusion (Penn class): } \\
\hline Penn A (absent) & $20(66.6 \%)$ \\
\hline Penn B (branch malperfusion) & $10(33.3 \%)$ \\
\hline Cerebral & $1(3.3 \%)$ \\
\hline Renal & $6(20 \%)$ \\
\hline Lower limb & $3(10 \%)$ \\
\hline \multicolumn{2}{|l|}{ Pre-operative echo data: } \\
\hline Ejection fraction EF 50-60\% & $27(90 \%)$ \\
\hline $\mathrm{EF}<40 \%$ & $3 \quad(10 \%)$ \\
\hline Moderate-severe aortic regurge & $23(76.6 \%)$ \\
\hline Pericardial effusion & $4 \quad(13 \%)$ \\
\hline
\end{tabular}

Of these 30 patients, there were 23 males and 7 females. The patient age range 30 to 70 years. 8 patients had Marfan syndrome and 2 patients had Bicuspid aortic valve disease. 20 patients were Penn class A and 10 Penn class B. All required emergency operation.

Operative data: (Table 2): Different surgical techniques were employed according to aortic pathology. 13 patients had modified Bentall procedure, 13 patients had supra coronary conduit replacement of ascending aorta, 4 patient had David reimplantation procedure.

Table (2): Operative data.

\begin{tabular}{ll}
\hline & $\begin{array}{c}\text { Number }(\%) \text { OR } \\
\text { Mean } \pm \text { SD }\end{array}$ \\
\hline Timing of operation: & \\
Elective & $30(100 \%)$ \\
Emergency & \\
Arterial inflow: & $25(83.3 \%)$ \\
Axillary artery & $5(16.6 \%)$ \\
Femora artery & $165 \pm 20$ \\
CPB duration (minutes) & $145 \pm 25$ \\
Cross clamp duration (minutes) & \\
Total circulatory arrest: TCA: & $25 \pm 10 \mathrm{mins}$ \\
TCA time (min) & \\
Cerebral perfusion: & $25(83.3 \%)$ \\
Antegrade (ACP) & $5(16.6 \%)$ \\
Retrograde (RCP) & \\
Coronary reimplantation/reattachment: & \\
Direct button & $11(36.3 \%)$ \\
Modified Cabrol & $3(10 \%)$ \\
SVG to RCA & $3(10 \%)$ \\
None & $13(43.3 \%)$ \\
Root surgery: & \\
Bentall procedure & $13(43 \%)$ \\
Supracoronary with sinus replacement & $13(43 \%)$ \\
Tirone David I & $4(14 \%)$ \\
\hline
\end{tabular}

- Cannulation sites for Cardiopulmonary Bypass (CPB): Arterial inflow was done through the femoral artery in 5 patients $(16.6 \%)$ and through the axillary artery in 25 patients $(83.3 \%)$.

- The mean cardiopulmonary bypass time was $165 \pm 20 \mathrm{~min}$ and The mean cross clamp time was $145 \pm 25 \mathrm{~min}$.

- Mode of coronary reimplantation and proximal reattachment: Direct button in 11 patients (36.3\%), modified cabrol in $3(10 \%)$, saphenous vein to right coronary artery in $3(10 \%)$.

- Antegrade cerebral protection was done in 25 patients and retrograde cerebral protection in 5 patients. 
Post-op data: (Table 3): In the ICU, the haemodynamic parameters, duration of inotropic support, duration of mechanical ventilation, amount of blood transfusion were recorded. In addition other data of the post-operative course were collected including total hospital stay, occurrence of major adverse events and operative mortality (death within 30 days post-operatively or during the same hospitalization). Major adverse events were defined by new renal impairment (serum creatinine levels $>2 \mathrm{mg} / \mathrm{dl}$ or twice the pre-operative creatinine), post-operative myocardial infarction (CK-MB levels $>125 \mathrm{IU} / \mathrm{l}$ during the first 72 hours), and post-operative stroke (new neurological deficit persisting more than 24 hours).

Regarding post-operative complications, two patients needed re-exploration due to excessive post-operative bleeding, 4 patients $(13.2 \%)$ were complicated by anuria and acute renal failure that required dialysis. These 4 patients were Penn class $\mathrm{B}$ with pre-operative renal malperfusion.

Table (3): Post-operative data.

\begin{tabular}{ll}
\hline & $\begin{array}{c}\text { Number (\%) OR } \\
\text { Mean } \pm \text { SD }\end{array}$ \\
\hline Mechanical ventillation duration (hours) & $12-72$ \\
ICU stay duraion (hours) & $84 \pm 12$ \\
Hospital stay duration (days) & $13.2 \pm 3.6$ \\
Blood loss & $1500 \pm 400 \mathrm{cc}$ \\
Re-exploration for bleeding & $2(6.6 \%)$ \\
Acute renal failure requiring dialysis & $4(13.2 \%)$ \\
CVA/stroke & $2(6.6 \%)$ \\
Mortality & $5(17 \%)$ \\
\hline
\end{tabular}

There was $5(17 \%)$ post-operative mortality: 1 due to massive bleeding, 2 due to prolonged ventilation following stroke, 1 due to hepato-renal failure and 1 due to myocardial failure. Mortality was strongly correlated with the pre-operative malperfusion status.

\section{Discussion}

Surgical outcomes of Acute Type A Aortic Dissection (ATAAD) are greatly influenced by preoperative patient status, particularly haemodynamic shock and organ ischaemia. In this context, several reports have addressed the impact of pre-operative malperfusion and ischaemia at presentation on post-operative results [3].

Appropriate evaluation of pre-operative patient clinical presentation may be key in determining the optimal surgical strategy and post-operative care and in predicting surgical outcome [5].

Patients with ATAAD and malperfusion have been considered by some groups for delayed sur- gery and interval endovascular intervention. In a study by Patel et al., patients with peripheral malperfusion underwent preoperative percutaneous fenestration and stenting of the aortic true lumen, with or without branch vessel stenting where appropriate. One-third of patients died before this could be done, and of the patients successfully fenestrated, $26 \%$ died prior to surgical replacement of ascending aorta [6]

In contrast, Perera et al., concluded that endovascular treatment of mesenteric malperfusion, when performed as a staged procedure before or after open central aortic repair, improves outcome in ATAAD. However, this strategy is not always feasible in cases where the acuity of the dissection necessitates immediate surgery or due to unavailability of a hybrid operating theatre in many centers [7].

Despite the advancement of cardiac surgery techniques, employment of modern cerebral protection strategies, myocardial protection, and aortic graft development, the surgical mortality of acute dissection operations, even in centers with specific interest in aortic surgery, is in the range of $25 \%$ Operative mortality is multifactorial, hemorrhage, cerebral ischemia, and distal malperfusion are leading causes [8].

It has been reported that as many as $20-30 \%$ of patients presenting with type A dissection may have one or more malperfusion syndromes.

Visceral and renal malperfusion are often under recognized at the time of presentation, with the emphasis placed on reconstructing the threatened proximal thoracic aorta and the associated aortic insufficiency. Distal malperfusion may manifest as ileofemoral, mesenteric, renal, cerebral or spinal cord in nature. The resulting lower limb ischemia, gut ischemia, renal failure and stroke significantly increases mortality [9].

Our study carried out during the period between 2012 and 2014 on 30 consecutive ATAAD patients, there were 23 males and 7 females. The patient age range 30 to 70 years. 8 patients had Marfan syndrome and 2 patients had Bicuspid aortic valve disease. 20 patients were Penn class A without malperfusion and 10 Penn class B with renal, cerebral, and lower limb ischemia. All required emergency operation.

As regard pre-operative degree of aortic regurge, $23(76.6 \%)$ out of the 30 patients had moderate to severe aortic regurge. 
Arterial inflow was done through the femoral artery in 5 patients $(16.6 \%)$ and through the axillary artery in 25 patients $(83.3 \%)$. The mean cardiopulmonary bypass time was $165 \pm 20 \mathrm{~min}$ and the mean cross clamp time was $145 \pm 25 \mathrm{~min}$. 13 patients had modified Bentall procedure, 13 patients had supra coronary conduit replacement of ascending aorta, 4 patient had David reimplantation procedure. Coronary reimplantation was by Direct button in 11 patients $(36.3 \%)$, modified cabrol in $3(10 \%)$, saphenous vein to right coronary artery in $3(10 \%)$. Antegrade cerebral protection was done in 25 patients and retrograde in 5 patients.

Re-exploration for bleeding was needed in 2 patients, 4 patients $(13.2 \%)$ were complicated by acute renal failure that required dialysis. These 4 patients were Penn class B with preoperative renal malperfusion.

There was $5(17 \%)$ post-operative mortality: due to massive bleeding, 2 due to prolonged ventilation following stroke, 1 due to hepato-renal failure and 1 due to myocardial failure. Mortality was strongly correlated with the pre-operative malperfusion status.

Danielson and colleagues 2015 [3] reviewed 341 consecutive patients surgically treated for ATAAD between 1998 and 2014 recruited for retrospective observational study. In-hospital mortality rates by Penn class were as follows: Class A, $11 \%$; Class B, $14 \%$; Class C, $42 \%$. The leading cause of late mortality was cardiac failure or myocardial infarction (29\%), followed by aortic rupture $(25 \%)$. Penn classes $\mathrm{c}$ and $\mathrm{bc}$ were identified as an independent risk factor for in-hospital mortality. Regardless of ischaemic manifestations at presentation, the prognosis of patients surviving both surgery and early post-operative period proved acceptable [3].

\section{Conclusion:}

- Aortic dissection surgery is always challenging and never straightforward. Special considerations required for all patients include proper preoperative imaging, proper myocardial protection, proper Cardio-Pulmonary Bypass (CPB) strategy.

- The overall short term survivals is greatly affected by malperfusion syndromes at initial presentation.
Study limitations:

The present study is limited by its retrospective and observational design, as well as the small sample size. Bigger number of patients with different malperfusion syndromes should be compared for better evaluation of outcome of acute dissection surgery.

\section{References}

1- HAGAN P.G., NIENABER C.A., ISSELBACHER E.M., BRUCKMAN D., KARAVITE D.J., RUSSMAN P.L., et al.: The International Registry of Acute Aortic Dissection (IRAD): New insights into an old disease. J.A.M.A., Vol. 283: 897-903, 2000

2- BOOHER A.M., ISSELBACHER E.M., NIENABER C.A., TRIMARCHI S., EVANGELISTA A., MONTGOMERY D.G., et al.: The IRAD classification system for characterizing survival after aortic dissection. Am. J. Med., 126: 730 e 19-24, 2013.

3- DANIELSSON E., ZINDOVIC I., BJURSTEN H., INGEMANSSON R., and NOZOHOOR S.: Generalized ischaemia in type A aortic dissections predicts early surgical outcomes only. Interactive CardioVascular and Thoracic Surgery, 21: 583-9, 2015.

4- OLSSON C., HILLEBRANT C.G., LISKA J., LOCKOWANDT U., ERIKSSON P. and FRANCO-CERECEDA A.: Mortality in acute type A aortic dissection: Validation of the Penn classification. Ann. Thorac. Surg., 92: 1376$82,2011$.

5- AUGOUSTIDES J.G., GEIRSSON A., SZETO W.Y., WALSH E.K., CORNELIUS B., POCHETTINO A., et al.: Observational study of mortality risk stratification by ischemic presentation in patients with acute type A aortic dissection. Nat. Clin. Pract. Cardiovasc. Med., 6: 140-6, 2009.

6- PATEL H.J., WILLIAMS D.M., DASIKA N.L., SUZUKI Y. and DEEB G.M. : Operative delay for peripheral malperfusion syndrome in acute type A aortic dissection: A long-term analysis. J. Thorac. Cardiovascular. Surg., 135: 1288-96, 2008.

7- PERERA N.K., GALVIN S.D., SEEVANAYAGAM S and MATALANIS G.: Optimal management of acute type A aortic dissection with mesenteric malperfusion. Interact. Cardio. Vasc. Thorac. Surg., 19: 290-4, 2014.

8- APPOO J.J. and POZEG Z.: Strategies in the surgical treatment of type A aortic arch dissection. Ann. Cardiothorac. Surg., 2 (2): 205-11, 2013.

9- PACINI D., LEONE A., BELOTTI L.M., et al.: Acute type A aortic dissection: Significance of multiorgan malperfusion. Eur. J. Cardiothorac. Surg., 43: 820-6, 2013. 


\section{العوامل الإكلينيكية والتشخيصية ما قبل الجراحية المنبئة

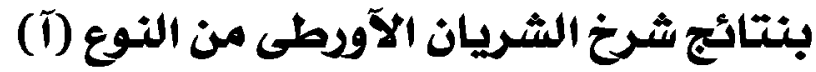

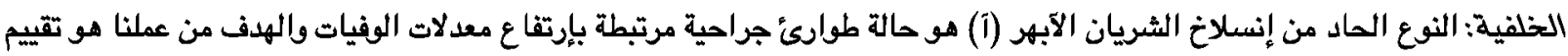

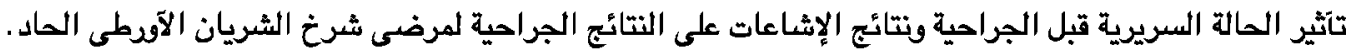

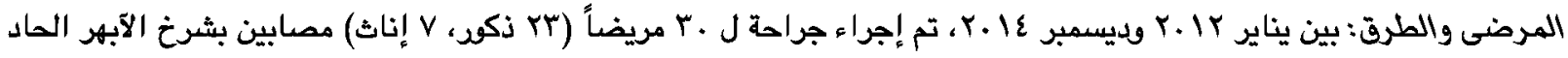

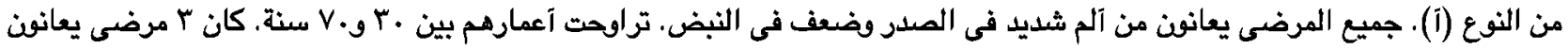

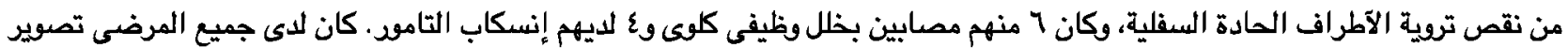

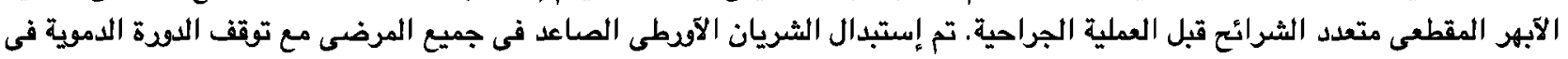

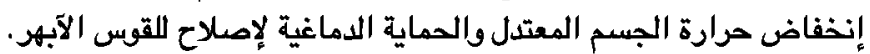

النتائج: كان هناك ه (IV) وفيات ما بعد الجراحة: البسبب نزيف حاد، ب بسبب السكةة الدماغية، ا بسبب الفشل الكلوى ول بسبب فشل عضلة القلب.

الخلاصدة: العلاج الجراحى لتشرح الآبهر الحاد هو تحدى ويتطلب إستراتيجية جراحية جيدة جيدة للحصول على التيجة المثلى. المرضى

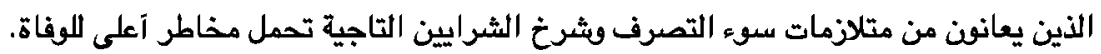

